Revue internationale P.M.E.

Économie et gestion de la petite et moyenne entreprise

\title{
PME en croissance : peut-on prévoir les seuils organisationnels?
}

\section{Armelle Godener}

Volume 15, numéro 1, 2002

URI : https://id.erudit.org/iderudit/1008800ar

DOI : https://doi.org/10.7202/1008800ar

Aller au sommaire du numéro

\section{Éditeur(s)}

Presses de l’Université du Québec

ISSN

0776-5436 (imprimé)

1918-9699 (numérique)

Découvrir la revue

Citer cet article

Godener, A. (2002). PME en croissance : peut-on prévoir les seuils organisationnels ? Revue internationale P.M.E., 15(1), 39-63. https://doi.org/10.7202/1008800ar

\section{Résumé de l'article}

Le mode d'organisation des entreprises en croissance est réputé évoluer dans l'urgence et dans la crise, après la survenue d'un seuil organisationnel, révélateur tardif de l'arrivée à saturation du mode d'organisation en place. La recension de la documentation existante concluant à l'absence d'informations opérationnelles en la matière, l'auteure met en avant l'intérêt de recherches visant à caractériser le moment de survenue de ces seuils sous une forme permettant à tout dirigeant de PME en croissance de prévoir leur survenue prochaine et, donc, de s’y préparer. À titre exploratoire, une étude terrain, fondée sur l'histoire de 23 PMI en croissance, a permis de mettre en évidence que ces seuils semblent surgir systématiquement dans le sillage de transformations radicales de la firme, également liées à sa croissance, qui joueraient un rôle de catalyseur. 


\title{
PME en croissance: peut-on prévoir les seuils organisationnels?
}

\author{
Armelle GODENER \\ École supérieure de commerce de Grenoble
}

\section{MOTS CLÉS}

\section{PME - Croissance - Organisation}

\begin{abstract}
RÉSUMÉ
Le mode d'organisation des entreprises en croissance est réputé évoluer dans l'urgence et dans la crise, après la survenue d'un seuil organisationnel, révélateur tardif de l'arrivée à saturation du mode d'organisation en place. La recension de la documentation existante concluant à l'absence d'informations opérationnelles en la matière, l'auteure met en avant l'intérêt de recherches visant à caractériser le moment de survenue de ces seuils sous une forme permettant à tout dirigeant de PME en croissance de prévoir leur survenue prochaine et, donc, de s'y préparer. À titre exploratoire, une étude terrain, fondée sur l'histoire de 23 PMI en croissance, a permis de mettre en évidence que ces seuils semblent surgir systématiquement dans le sillage de transformations radicales de la firme, également liées à sa croissance, qui joueraient un rôle de catalyseur.
\end{abstract}

\begin{abstract}
The organization of growing companies is said to be transformed in emergency and through crisis period, after the arrival of an organizational "threshold", which reveals the saturation of the current organization. The review of existing literature concluding to a lack in operational information in that matter, the author points out the interest of surveys aiming to characterize the very moment when these thresholds appear
\end{abstract}

\section{L'AUTEURE}

ARMELLE GodenER est professeure associée de comptabilité analytique et de contrôle de gestion au Groupe École supérieure de commerce de Grenoble qu'elle a rejoint après avoir exercé pendant six ans en entreprise la fonction de contrôleure de gestion. Ses recherches se focalisent sur le contrôle de gestion, le management de la R-D et les crises de croissance des PME. Cet article est issu de son travail doctoral, réalisé sous la direction de Robert Paturel, Cerag - ESA Grenoble. Adresse : 12, rue Pierre-Semard, 38003 Grenoble Cedex 03, France. Courriel : <armelle.godener@esc-grenoble.fr>. 
in a way allowing every growing SME's CEO to foresee them and then to prepare the mutation. On an exploratory basis, a field study, based upon the evolution of 23 medium and small companies, showed that these thresholds seem to occur systematically at the very moment or just after predictable radical changes.

\section{RESUMEN}

El modo de organización de las empresas en crecimiento está previsto que evolucione en un ámbito de urgencia y de crisis, consecutivamente a la emergencia de un umbral organizativo, revelador tardío de la llegada a saturación del modo de organización vigente. El examen de la literatura existente concluyendo en la ausencia de informaciones operativas en la materia, el autor subraya el interés de las investigaciones dirigidas a caracterizar el momento en que estos umbrales se presentan, bajo una forma que permita a cualquier directivo de una PyME en crecimiento, prever su llegada inminente y, en consecuencia, de prepararse a ello. Un estudio de terreno exploratorio, fundado sobre la historia de veintitrés PyMEs en crecimiento, ha permitido poner en evidencia que estos umbrales parecen surgir sistemáticamente arrastrados por la dinámica de transformaciones radicales de la firma, vinculadas asimismo a su crecimiento, lo cual desempeñaría un papel de catalizador.

\section{ZUSAMMENFASSUNG}

Die Organisationsform der wachsenden Unternehmen entwickelt sich bekanntlich aus dem Notfall heraus und in der Krise, als eine Folge eines plötzlichen Erscheinens einer spät entdeckten Organisationsschwelle. Die Durchsicht der existierenden Literatur lässt den Schluss zu, dass die Forschung versucht, den Moment der auftauchenden Organisationsschwelle zu umschreiben. Die Kenntnis dieser Schwelle erlaubt für jeden Unternehmensleiter von wachsenden KMU, die auftauchenden Probleme vorherzusehen und entsprechend zu reagieren. Eine Untersuchung wurde bei 23 wachsenden, industriellen KMU durchgeführt, welche klar aufzeigte, dass solche Entwicklungsstufen systematisch vor radikalen organisatorischen Veränderungen auftauchen.

\section{Introduction}

À la fin des années 1980, 50\% des managers suédois qui estimaient possible une expansion durable et profitable de leur entreprise déclaraient ne pas chercher à exploiter cette opportunité (Davidsson, 1989). C'est que la croissance ${ }^{1}$, quel que soit le mode retenu - croissance interne ou croissance externe -, est une source importante de difficultés et de risques de tous ordres pour la petite et moyenne entreprise : risques commerciaux, dangers financiers, difficultés liées à la nécessaire mutation du management, perte potentielle de pouvoir, etc. (Girard, 1997).

1. Définie traditionnellement par «des chiffres d'affaires qui augmentent, des actifs qui s'accumulent, des effectifs qui se gonflent» (De Montmorillon, 1989). 
Dans le cadre spécifique de la croissance interne de la petite et moyenne entreprise, les difficultés peuvent provenir de l'environnement externe de la firme. Il s'agit alors de barrières au maintien sur les marchés traditionnels de l'entreprise considérée (développement de la concurrence, évolution technologique mettant en cause les savoir-faire de la firme et ses actifs productifs, etc.), de barrières à la croissance sur ces mêmes marchés (saturation du marché, technologie imposant des augmentations brutales de capacité, ressources rares dont la pénurie s'accroît, etc.), ou encore de barrières à l'entrée des domaines d'activité stratégique convoités. Les entreprises concernées par notre recherche ont surmonté, sur une période significative, les éventuels obstacles de cette catégorie qu'elles ont rencontrés.

Elles sont parvenues, de plus, à surmonter ou à éviter des faiblesses ou des erreurs qui peuvent constituer, ponctuellement, des obstacles majeurs à la croissance, voire à la survie : échec d'une tentative de lancement d'un nouveau produit, défaillance d'un client essentiel, baisse de compétitivité, problème de succession du dirigeant, etc.

Il leur reste alors à surmonter une dernière catégorie d'obstacles, pour lesquels nous avons retenu le nom générique de «seuils à franchir » et dont les modèles de métamorphose enseignent qu'ils apparaissent inéluctablement dès lors que la croissance se prolonge suffisamment longtemps. Ils correspondent à la saturation d'au moins un organe de l'entreprise et leur survenue impose une « révolution » interne, généralement qualifiée de critique.

Dans une première partie, nous montrerons que, si les modèles de métamorphose stipulent leur existence et décrivent de quelle façon ils s'opposent à la poursuite de la croissance, leur connaissance mérite d'être complétée afin que les dirigeants concernés puissent véritablement préparer le changement et, peut-être, limiter ainsi l'intensité des traditionnelles crises de croissance (section 1). Notre étude terrain est une recherche exploratoire allant dans ce sens (sections 2 et 3).

\section{Les changements de dimension organisationnelle : des périodes de mutation considérées comme critiques}

Dans le cadre des modèles de métamorphose ${ }^{2}$, chercheurs et consultants se sont attachés à établir des portraits robots de «l'entreprise type » tout au long de sa

2. Certaines publications entrant dans ce champ sont fondées sur la théorie du cycle de vie de l'entreprise (la croissance de la firme est alors considérée comme un phénomène naturel et inéluctable, faisant suite à la période de création et précédant une période de maturité, puis de déclin) quand d'autres ne font aucune référence à ce sujet. Par leurs descriptions relatives aux périodes d'expansion, ces deux catégories de modèles se complètent dès lors qu'il est fait abstraction de cette éventuelle hypothèse de base. Nous ne distinguons donc pas les uns et les autres dans notre discussion.

Revue internationale P.M.E., vol. 15, nº 1, 2002 
croissance éventuelle. Ils décrivent ainsi de trois à cinq stades de développement, depuis celui de la petite entreprise aux capitaux exclusivement familiaux à la structure informelle et à l'activité unique jusqu'à celui de la grande entreprise au capital ouvert à de nouveaux partenaires, à la structure formalisée et décentralisée, avec une activité diversifiée et/ou une présence conséquente sur des marchés internationaux.

Or, à travers l'étude de la vie de 110 petites et moyennes entreprises en croissance, Churchill et Lewis (1983) ont montré que le schéma des modèles de métamorphose doit être compris comme une tendance générale d'évolution plutôt que comme une vérité universelle. En particulier, ils ont observé qu'une entreprise peut se situer «à un certain stade à l'endroit de certains facteurs et à un autre concernant des facteurs différents ». Ce constat impose d'étudier les stades de développement et les périodes de passage d'un stade à l'autre, non pas comme si elles concernaient systématiquement la totalité des caractéristiques de l'entreprise, mais en se focalisant sur un organe donné. Dans cette logique, nous nous sommes intéressée plus particulièrement aux apports des modèles de métamorphose relatifs à l'évolution du mode d'organisation de la firme en croissance.

Nous faisons d'abord le point de la connaissance établie par rapport au processus générateur de changement (1.1), puis présentons comment la mutation est réputée entreprise dans un contexte de crise (1.2) afin de mettre en évidence en quoi ce schéma mérite d'être complété en vue de permettre aux dirigeants de l'intégrer dans leur prise de décision stratégique.

\subsection{Une évolution généralement entreprise sous la contrainte}

Les modèles de métamorphose ${ }^{3}$ présentent la transformation du mode de fonctionnement de la firme en croissance comme un processus discontinu. Des périodes de développement des ventes et de relative stabilité du fonctionnement interne (que nous désignons par l'expression « stades de développement ») sont séparées les unes des autres par des périodes de mutations internes profondes (appelées ici «changements de dimension organisationnelle»). Chaque période de mutation est réputée n'être entreprise que sous la contrainte. Un coup d'œil rapide au schéma proposé par les modèles de métamorphose montre combien la pression sur le dirigeant de

3. Les différents modèles se complétant les uns les autres, nous proposons ici une synthèse des écrits de Filley et House (cités par Gasse et Carrier, 1992), Steinmetz (1969), Salter (1970), Greiner (1972), Basire (1976), Brac de la Perrière (1978), Churchill et Lewis (1983), Miller et Friesen (1984), Parent (1985), Smith, Mitchell et Summer (1985) et Adizes (1991). 
l'entreprise est alors importante et comment apparaissent de cruciales difficultés financières pouvant conduire à l'arrêt de la croissance, voire à la disparition de la firme (par arrêt de l'activité ou absorption par une autre société).

\subsubsection{Le premier stade de développement}

Une fois passée la phase de création, le premier stade de développement correspond à la période durant laquelle l'entreprise est généralement gérée quasi exclusivement par son dirigeant, véritable homme à tout faire. Celui-ci participe aussi bien à la fonction de production qu' à celle de vente, ces deux activités correspondant aux deux postes clés auxquels il doit porter toute son attention. En revanche, il ne remplit pas de fonction de management à proprement parler. L'entreprise se satisfait d'une organisation informelle et d' une faible spécialisation des fonctions. Chacun trouve sa place et son rôle selon les besoins du moment. La proximité géographique, conjuguée à la taille restreinte de l'effectif, permet à l'information de circuler naturellement au sein de l'ensemble du personnel. La convivialité est de mise.

Mais, si la croissance continue, l'équilibre ainsi établi ne peut être maintenu indéfiniment. C'est que, pour faire face aux tâches administratives qui se multiplient (facturation, déclarations fiscales et sociales, etc.) ou pour respecter les délais de livraison garantis aux clients, du personnel supplémentaire est progressivement embauché. Du fait de cette augmentation de l'effectif, la supervision de chacun, la coordination et la communication entre services finissent par ne plus pouvoir être assurées de façon informelle. L'ajustement mutuel a atteint ses limites et de sérieux dysfonctionnements (goulots d'étranglements, non-respect des délais de livraison, baisse de la qualité des produits, retards de facturation, etc. ${ }^{4}$ ) apparaissent et provoquent, tant que l'organisation ne se remet pas en cause, une dégradation rapide de la situation financière. En tentant de régler les problèmes à mesure qu'ils se posent, le dirigeant se trouve submergé. Les vingt-quatre heures d'une journée ne lui suffisent pas pour effectuer l'ensemble des tâches quotidiennes qui lui incombent. Toute prise de recul et toute décision dont la portée n'est pas immédiate sont reportées à plus tard, ce qui grève d'autant la capacité de croissance de la firme.

Selon le schéma établi, ce n'est qu'alors qu'est engagé le premier changement de dimension organisationnelle qui conduit la firme vers le deuxième stade de développement.

\subsubsection{Le deuxième stade}

À ce stade, la structure de l'entreprise type a été précisée et la spécialisation des tâches développée. Des responsables ont été nommés à la tête des principales

4. Steinmetz (1969), Gasse et Carrier (1992).

Revue internationale P.M.E., vol. 15, nº 1, 2002 
fonctions et la structure est dite de type fonctionnel. La communication est devenue plus impersonnelle et plus formelle. Un système d'information a été mis en place, aussi bien pour surveiller l'environnement que pour favoriser la communication entre les services, dans une perspective de coordination des actions. D'homme à tout faire, le dirigeant est ainsi devenu superviseur. Il ne délègue pour autant que la gestion courante, une grande centralisation étant de rigueur.

La croissance de la firme peut alors redémarrer dans de bonnes conditions... jusqu'à ce que la structure en place montre à son tour des signes évidents de saturation. Si la croissance se poursuit assez longtemps, vient en effet, selon les modèles de métamorphose, le jour où le dirigeant se trouve à nouveau totalement engagé dans des opérations de court terme et confronté à un manque chronique de temps. Des décisions sont alors prises trop vite. Certaines divisions sont laissées sans direction et la coordination, que le chef d'entreprise assure traditionnellement, perd de son efficacité. Une baisse de la qualité des produits et des services rendus, une détérioration du chiffre d'affaires et des coûts puis des problèmes de trésorerie sont réputés suivre alors 5 .

Pour que la croissance puisse reprendre, voire pour la survie de la firme, un nouveau changement de dimension organisationnelle est alors entrepris. Cette période de mutation ouvre la porte au troisième stade de développement, celui de la décentralisation.

\subsubsection{Le troisième stade}

De nouveaux niveaux hiérarchiques sont maintenant créés. La délégation est réelle et s'exerce soit par unité géographique, soit par division. Smith, Mitchell et Summer (1985) relèvent que les dirigeants prennent leurs décisions en tenant compte de l'avis de leurs subordonnés et recherchent leur adhésion, ce qui n'était pas le cas précédemment. Pour le dirigeant, il s'agit d'une révision profonde de son métier et de son comportement. Le système de contrôle s'est étoffé et sophistiqué et la gestion du personnel se développe encore avec un élargissement de sa mission et la formalisation du système de récompenses.

Mais la poursuite éventuelle de la croissance entraîne, à terme, l'entreprise vers de nouveaux déséquilibres (et de nouvelles possibilités) en termes d'organisation et exige de nouvelles mutations. Nous ne poursuivrons pourtant pas cette synthèse des modèles de métamorphose au-delà de ce troisième stade, notre centre d'intérêt étant celui de la petite et moyenne entreprise.

Ainsi, selon les modèles de métamorphose, la petite et moyenne entreprise en croissance est réputée n'engager une transformation de son mode d'organisation

5. Basire (1976), Tashakori (1980), Hofer et Charan (1984), Flamholtz (1986).

Revue internationale P.M.E., vol. 15, nº 1, 2002

(C) 2002 - Presses de l'Université du Québec

Édifice Le Delta I, 2875, boul. Laurier, bureau 450, Sainte-Foy, Québec G1V 2M2 • Tél. : (418) 657-4399 - www.puq.uquebec.ca

Tiré de : Revue internationale P.M.E., vol. $15, \mathrm{n}^{\circ} 1$, sous la direction de Pierre-André Julien. 
que lorsque survient ce que nous proposons d'appeler un seuil organisationnel, défini comme le moment ultime où le dirigeant de la firme considérée perçoit, sous la pression des dysfonctionnements, qu'il est temps d'engager un nouveau changement de dimension organisationnelle. La figure 1 synthétise la succession d'étapes ainsi décrites en intégrant les seuils organisationnels au célèbre schéma de Greiner.

FIGURE 1

Les différentes phases d'évolution du mode d'organisation de la petite et moyenne entreprise en croissance

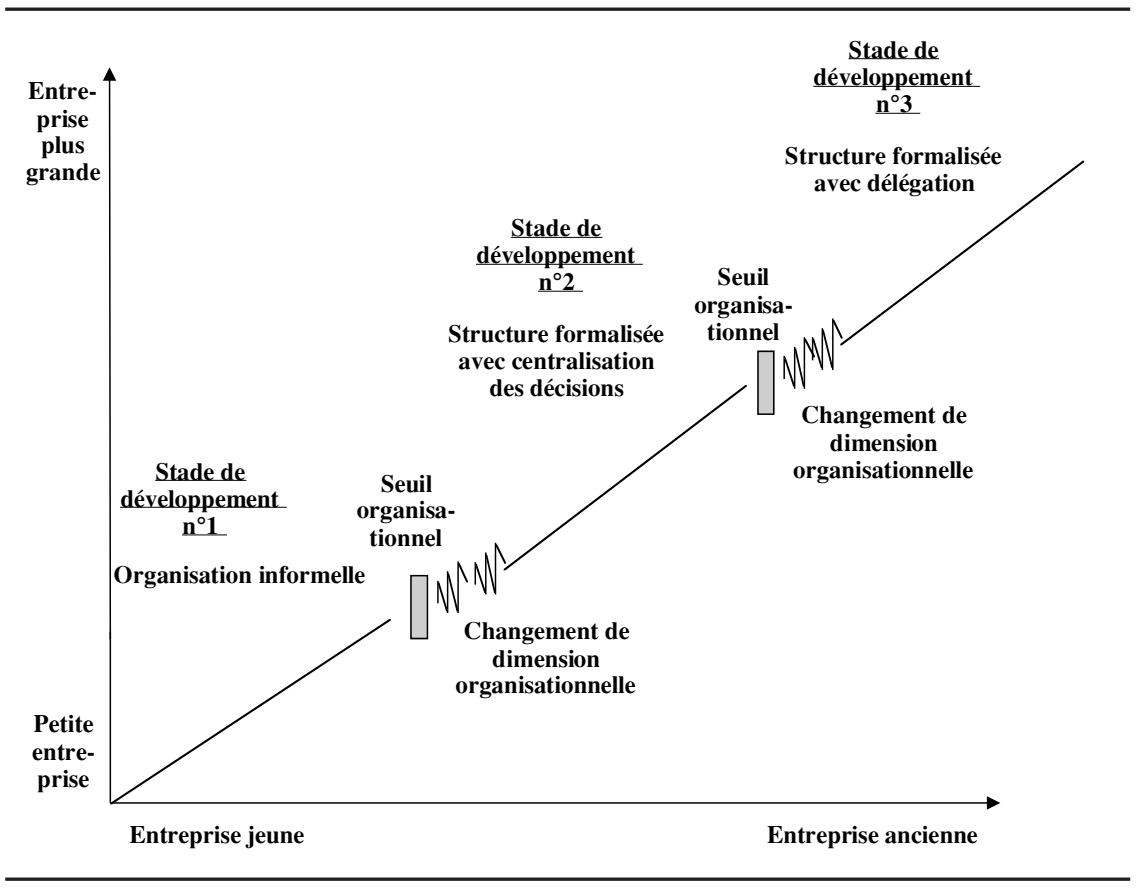

Source : Schéma inspiré de Greiner (1972).

Mais les difficultés liées à l'apparition du seuil ne sont pas passagères. La période de mutation elle-même amène son lot de perturbations.

\subsection{Le caractère critique des changements de dimension}

Consultants ou chercheurs, les auteurs s'accordent pour présenter les périodes de changements de dimension organisationnelle comme des périodes de crise, c'est-à-dire des périodes de tensions extrêmes à l'issue incertaine. 
Les difficultés rencontrées dans ces moments décisifs pour la poursuite de la croissance, voire la survie de la firme ${ }^{6}$ sont en effet nombreuses. En premier lieu, l'identification des causes profondes de la crise est délicate puisque la nature diverse des symptômes (malaise important au sein de la firme, insatisfaction des clients et donc baisse du chiffre d'affaires et dégradation financière, etc.) risque d'orienter les acteurs de la firme vers de fausses pistes. De plus, admettre la nécessité de transformer profondément le mode d'organisation en vigueur, changer d' habitudes et de comportement sont des impératifs difficiles à vivre pour le dirigeant comme pour les responsables nouvellement nommés et l'ensemble du personnel qui doit apprendre à vivre dans le cadre de nouveaux jeux de pouvoir. Enfin, il n'est pas toujours aisé d'attirer des cadres formés dans de grandes entreprises et disposant des savoir-faire et savoir-être requis (Girard, 1997).

Sur ces points, les apports des modèles de métamorphose (qui décrivent tant les changements de dimension à mener que les symptômes de crise) comme les écrits relatifs à la conduite du changement constituent autant d'outils susceptibles de faciliter la mutation à entreprendre.

Mais nous pensons que la situation se trouve encore aggravée lorsque - et si l'on en croit la littérature, c'est la tendance générale - la transformation de la firme n'est engagée qu'après l'apparition (et l'interprétation correcte) des symptômes de crise correspondant au seuil organisationnel. En effet, pour salutaire que puisse être une crise en tant qu'événement mobilisateur des énergies ${ }^{7}$, agir dans l'urgence et en période de tension extrême est une situation inconfortable qui accroît les risques d'échec. Le dirigeant, débordé par les problèmes à résoudre pour satisfaire la clientèle et soutenir coûte que coûte la performance de la firme, ne peut que difficilement prendre du recul pour décider d'une solution et tout faire pour la mettre en œuvre. De plus, l'adoption d'un nouveau cadre de référence ne peut être instantané. Les acteurs concernés par le changement, dirigeant compris, doivent disposer d'un délai pour s'approprier l'idée de la solution envisagée, puis pour la mettre en œuvre : faire confiance et déléguer pour les uns, prendre des décisions et assumer des responsabilités nouvelles pour les autres, requièrent un apprentissage, de nouvelles habitudes, de nouveaux repères. Un changement de comportement ne se décrète pas, mais se construit par la pratique et donc dans la durée. Ainsi, nous estimons qu' un minimum d'anticipation des seuils organisationnels est souhaitable, car de nature à limiter les difficultés et, par là, à permettre d'éviter entre autres les difficultés financières et les risques encourus.

6. Steinmetz (1969), Greiner (1972) et Churchill et Lewis (1983) sont particulièrement explicites quant aux risques encourus par l'entreprise qui ne parviendrait pas à se transformer à temps.

7. Voir à ce sujet Greiner (1972, 1977), Brilman (1985, p. 26), Bartoli et Hermel (1986, p. 190-191).

Revue internationale P.M.E., vol. 15, $\mathrm{n}^{\circ}$ 1, 2002 
Pour autant, il importe de ne pas doter prématurément la petite et moyenne entreprise d'une structure alourdie en personnel d'encadrement ou en systèmes de communication. Gasse et Carrier (1992, p. 24-25) illustrent les dangers d'une telle attitude à travers une analogie à l'évolution de l'individu : « On sait que le fait d'imposer trop rapidement à un adolescent des exigences qui relèvent des adultes peut avoir pour conséquences le retard ou le ralentissement de son développement plutôt que sa progression. On peut affirmer qu'il en va de même pour l'entreprise [...]».

Les changements de dimension organisationnelle doivent donc être engagés au bon moment, ni trop tôt, ni trop tard.

Or, la littérature existante, qu'elle relève de la théorie des organisations ou des modèles de métamorphose, ne fournit pas d'information factuelle suffisamment précise pour que le dirigeant d'une petite et moyenne entreprise en croissance puisse identifier que le moment approche où il considérera comme impératif et urgent de transformer le mode d'organisation de sa firme, à la suite de la multiplication de dysfonctionnements. En effet, si plusieurs auteurs ont tenté d'indiquer au regard de chaque stade de développement les effectifs correspondants, les incohérences relevées d'une proposition à l'autre montrent que ce critère est insuffisant pour situer, pour une entreprise donnée, l'approche d'un seuil organisationnel. Ainsi, alors que pour Basire (1976) et Steinmetz (1969), le premier stade prend fin lorsque l'entreprise comprend de 25 à 30 salariés, Brac de la Perrière (1978) fixe la limite aux alentours de 50 personnes et Gélinier et Gaultier (1974) comme Kalika à la centaine de salariés. C'est que le développement de l'effectif n'est qu'un des éléments expliquant la nécessité d'évolution de la structure de la firme. Il a été démontré que la diversité géographique de ses activités, le nombre de produits ou de lignes de produits, le nombre de canaux de distribution, l'importance des technologies dans les procédés de production, leur vitesse d'évolution, l'industrie et son taux de croissance ${ }^{8}$ sont d'autres facteurs qui interviennent. Nous pensons que l'expérience du chef d'entreprise, sa capacité de travail, son sens du concret et / ou de la stratégie, ses qualités humaines, tout comme le niveau de qualification moyen du personnel sont autant de variables susceptibles de repousser un temps le moment de la survenue du seuil organisationnel.

Dans ce contexte, il apparaît comme pertinent d'engager des recherches visant à préciser le moment où surviennent fréquemment les seuils organisationnels en le caractérisant à partir d'éléments perceptibles (ou connus) à l'avance. Peuton trouver des points communs dans les situations précédant la survenue des seuils? Existe-t-il des régularités? Grâce à cette information, les dirigeants d'entreprise en croissance seraient plus à même de prendre conscience, ne serait-ce que peu de

8. Voir Woodward (1965), Salter (1970), Greiner (1972), Churchill et Lewis (1983), Miller et Friesen (1984).

Revue internationale P.M.E., vol. 15, $\mathrm{n}^{\circ} 1,2002$ 
temps à l'avance, qu'approche sans doute le moment d'entreprendre le passage au stade de développement suivant. L'effet de surprise et donc l'urgence du changement et, par là même, le caractère critique de cette période en seraient réduits.

La recherche présentée ici est une première étape allant dans cette voie.

\section{La méthodologie de recherche retenue}

L'approche retenue est une approche inductive qualitative, fondée sur des entretiens en face à face avec les dirigeants ${ }^{9}$ de 23 petites et moyennes entreprises industrielles en croissance ou ayant connu la croissance par croissance interne pendant au moins quatre ans après leur période de création.

\subsection{La constitution du terrain d'observation}

Toutes les entreprises retenues ont pour activité principale une activité industrielle et sont connues au plan régional ${ }^{10}$ pour leur croissance qui s'est prolongée au-delà de quatre ans après la période de création - et peut donc être considérée comme « durable». Cette croissance s'est systématiquement traduite par un développement des effectifs, du chiffre d'affaires et du capital.

Un premier contact téléphonique avec chacune des entreprises pressenties a permis de ne retenir que celles correspondant à quelques critères précis. En premier lieu, pour rester dans le cadre des modèles de métamorphose, nous n'avons retenu pour notre étude terrain que des entreprises évoluant par croissance interne. De même, afin de rester dans le cadre spécifique de la PME, nous n'avons travaillé que sur des entreprises que nous pouvons qualifier d'autonomes et stratégiquement indépendantes: sur les 23 firmes étudiées, 20 ont été créées par un individu en dehors de toute appartenance à un groupe; les trois autres, constituées par une entreprise industrielle existante, n'ont été retenues qu'après que leur dirigeant nous eut assuré bénéficier d'une indépendance de fait quant aux moyens disponibles et à la structure en vigueur depuis que leur entreprise a été effectivement créée ${ }^{11}$,

9. Dans quatre cas, l'indisponibilité du dirigeant a conduit à interroger l'un de ses proches collaborateurs qui, dans les quatre cas, s'est trouvé être le directeur administratif et financier.

10. Pour une question de facilité de contact, les firmes retenues font toutes partie de la région Rhône-Alpes qui constitue la deuxième région économique française et ont été détectées grâce à des intermédiaires bien introduits dans le milieu économique régional ou, pour certaines, grâce à un classement diffusé par la revue L'Entreprise.

11. Ces entreprises ne bénéficient ainsi ni d'apports en moyens (compétences, trésorerie, moyens techniques), ni de conseils (ou contraintes) en termes d'organisation, hormis un léger reporting financier peu contraignant.

Revue internationale P.M.E., vol. 15, nº 1, 2002 
ce qui les rend, de notre point de vue, comparables aux autres entreprises par rapport à notre problématique de seuils organisationnels. Leur évolution a été analysée jusqu'au moment de leur éventuel rachat par un groupe dont les conséquences figurent dans notre analyse.

Étant donné les différences d'ancienneté des sociétés et notre volonté de limiter nos observations aux périodes personnellement vécues par nos répondants, une grande disparité quant à la durée des tranches de vie disponibles pour notre analyse peut être observée. Dans certains cas, nous avons eu accès à l'intégralité de la période de croissance de l'entreprise et, dans d'autres, seulement à la période la plus récente. De plus, aucune limite du taux de croissance n'a été fixée. Certaines entreprises ont vécu une croissance très rapide (parfois plus de $20 \%$ par an sur plusieurs années), d'autres une évolution plus modérée (4\% à $5 \%$ par an).

Malgré ces disparités, les changements de dimensions et événements marquants évoqués par les répondants sont d'une très grande cohérence, ce qui nous permet d'en faire une analyse comparative.

Le tableau 1 synthétise les paramètres d'échantillonnage retenus et les tableaux 3 à 5 fournissent quelques éléments descriptifs des entreprises étudiées.

TABLEAU 1

Paramètres d'échantillonnage

\begin{tabular}{ll}
\hline $\begin{array}{l}\text { Paramètres } \\
\text { d'échantillonnage }\end{array}$ & Choix arrêtés \\
\hline Milieux & $\begin{array}{l}\text { Entreprises : petites et moyennes entreprises industrielles } \\
\text { ayant connu la croissance interne au-delà de leurs quatre premières } \\
\text { années d'existence. Ces entreprises sont connues régionalement } \\
\text { pour l'augmentation de leur activité. } \\
\text { Périodes retenues : depuis l'arrivée du répondant dans l'entreprise } \\
\text { jusqu'à la fin de la période de croissance, en dehors des périodes de } \\
\text { création (quatre premières années) et d'éventuelles périodes } \\
\text { d'opérations de croissance externe. }\end{array}$ \\
\hline Acteurs & \begin{tabular}{l} 
Dirigeants de l'entreprise ou, dans quelques cas, un de ses proches. \\
\hline Événements
\end{tabular} \\
\hline $\begin{array}{l}\text { Ruptures, transformations marquantes liées à la croissance interne, } \\
\text { en dehors de toute opération de croissance externe. }\end{array}$ \\
\hline Processus & $\begin{array}{l}\text { Ruptures, transformations, prises de décision, actions, } \\
\text { conséquences, contexte. }\end{array}$ \\
\hline
\end{tabular}

Source : Élaboré selon les indications de Huberman et Miles (1991, p. 64-65). 
TABLEAU 2

Effectifs des entreprises du terrain d'observation

\begin{tabular}{lcccccc}
\hline Employés & $\mathbf{0}$ à $\mathbf{1 5}$ & $\mathbf{1 5}$ à $\mathbf{5 0}$ & $\mathbf{5 0}$ à $\mathbf{1 0 0}$ & $\mathbf{1 0 0}$ à 200 & $\mathbf{> 2 0 0}$ & Total \\
\hline $\begin{array}{l}\text { Nombre d'entreprises } \\
\text { en début de période } \\
\text { étudiée }\end{array}$ & 7 & 8 & 3 & 4 & 1 & 23 \\
\hline $\begin{array}{l}\text { Nombre d'entreprises } \\
\text { en fin de période } \\
\text { étudiée }\end{array}$ & 0 & 4 & 7 & 8 & 4 & 23 \\
\hline
\end{tabular}

TABLEAU 3

Statut du dirigeant des entreprises étudiées (en début de période)

\begin{tabular}{lccccc}
\hline & $\begin{array}{c}\text { Propriétaire- } \\
\text { créateur }\end{array}$ & $\begin{array}{c}\text { Propriétaire- } \\
\text { héritier du } \\
\text { créateur }\end{array}$ & $\begin{array}{c}\text { Propriétaire } \\
\text { par achat } \\
\text { de la société }\end{array}$ & Salarié & Total \\
\hline Nombre d'entreprises & 10 & 8 & 2 & 3 & 23 \\
\hline
\end{tabular}

TABLEaU 4

Durée des périodes étudiées

\begin{tabular}{cccccc}
\hline & $<4$ ans & $\mathbf{4}$ à $\mathbf{1 0}$ ans & $\mathbf{1 0}$ à 20 ans & $>20$ ans & Total \\
\hline Nombre d'entreprises & 0 & 12 & 4 & 7 & 23 \\
\hline
\end{tabular}

Nous devons souligner que le mode opératoire retenu a d'office exclu de notre champ d'observation les entreprises pour lesquelles la survenue d'un seuil organisationnel s'est soldé par leur disparition en tant que telle, c'est-à-dire par leur dissolution ou par leur fusion avec une autre entreprise. Il s'est même trouvé qu'aucune entreprise retenue n'a échoué dans le franchissement des seuils organisationnels rencontrés, aucun retour à un stade de développement précédent n'ayant été signalé. En ce sens, les éléments qui ressortent de notre étude ne peuvent donc prétendre recouvrir l'ensemble des possibles, mais seulement une partie d'entre eux.

\subsection{Une approche perceptuelle fondée sur la mémoire}

Notre recherche étant de type inductif, nous avons eu recours à des questions très ouvertes, au cours d'entretiens semi-directifs. Les responsables interrogés ont ainsi été d'abord invités à raconter l'histoire de leur entreprise en décrivant son évolution au cours de la période de croissance. Étaient aussi bien évoquées les transformations d'ordre commercial que celles d'ordre financier, stratégique ou organisationnel. Ce 
panorama établi, nous revenions sur chaque transformation liée à la croissance et demandions au répondant de préciser le contenu de la mutation (ce qui a changé), son contexte (pourquoi ce changement a-t-il été entrepris ? pourquoi à ce momentlà ? quelle atmosphère a présidé à sa mise en œuvre ? etc.) et ses caractéristiques (durée de la mutation, mutation délibérée ou émergente, mutation progressive ou constituant une rupture, intensité des difficultés rencontrées, etc.). Ce déroulement d'entretien est tout à fait analogue à celui défini par Churchill et Lewis (1983) qui consiste à demander aux dirigeants d'évoquer «les stades ou étapes par lesquels leurs entreprises étaient passées, de définir les modifications majeures intervenues au cours de chaque stade et de décrire les faits ayant conduit à ces modifications ».

C'est donc le point de vue des acteurs qui a été privilégié, tant pour la facilité d'accès à l'information que pour l'intérêt qu'il représente. Cette perspective est préconisée par Van de Ven (1987) dans le cadre du management stratégique:

Sans observer un processus de changement depuis la perspective du manager, il devient difficile (si ce n'est impossible) pour un chercheur de comprendre la dynamique à laquelle sont confrontés les managers impliqués dans un effort de management stratégique et, par là même, de générer des résultats qui soient pertinents par rapport à la théorie et à la pratique du management stratégique.

Du fait de ce choix, l'approche retenue comporte un biais que nous avons dû accepter : la mémoire peut être sélective et exagérer ou minimiser l'importance de certains faits (Wolfe et Jackson, 1987). Nous avons cherché à limiter l'importance de ce biais lors de la collecte des données en incitant notre interlocuteur à toujours se resituer dans le passé et à préciser les faits. À propos de chaque transformation, la personne interrogée était ainsi invitée à préciser la situation de l'époque : «Aviez-vous alors conscience de cet élément? Qu'est-ce-qui vous a permis de comprendre ce besoin? » De relance en relance, des compléments d'information nous ont été apportés.

\subsection{Le traitement des données et l'identification des seuils}

Les données collectées ont été traitées conformément aux préceptes défendus par Strauss et Corbin (1994) dans le cadre de la Grounded Theory qui rejoint, par bien des aspects, les préconisations de Huberman et Miles (1991) en matière de traitement de données qualitatives. Comme l'indique le nom attribué à cette méthodologie, ses auteurs croient en la force des théories élaborées directement à partir des informations qui émanent du terrain, ce qui en fait une méthode typiquement qualitative et inductive. Elle est, de plus, particulièrement indiquée dans le cadre de l'étude de processus, grâce au double système de codage préconisé dont nous précisons ci-après l'application que nous en avons faite (Morse, 1994).

Revue internationale P.M.E., vol. 15, nº 1, 2002

(C) 2002 - Presses de l'Université du Québec

Édifice Le Delta I, 2875, boul. Laurier, bureau 450, Sainte-Foy, Québec G1V 2M2 • Tél. : (418) 657-4399 - www.puq.uquebec.ca

Tiré de : Revue internationale P.M.E., vol. $15, n^{\circ} 1$, sous la direction de Pierre-André Julien. 


\subsubsection{La détermination de catégories de changements de dimension organisationnelle}

En premier lieu, nous avons procédé à un " codage ouvert » systématique, dans le but d'identifier des faits similaires d'une entreprise à une autre. Les caractéristiques de chaque cas ont été relevées dans des matrices, et des «catégories» regroupant des phénomènes de même nature ont été élaborées. Deux de ces catégories correspondent à deux types de changement de dimension organisationnelle : la formalisation et le développement de la délégation.

La catégorie «formalisation de la structure » a été constituée de toutes les opérations de remise en cause, au sein de l'ensemble de l'entreprise ou de l'un de ses sous-ensembles, d'un mode de fonctionnement fondé sur l'ajustement mutuel, lorsque cette remise en cause a abouti à la définition des différents postes de travail, de leurs relations les uns avec les autres et des circuits d'information.

Pour leur part, tous les changements de dimension regroupés sous l'appellation « développement de la délégation » correspondent à un transfert, perçu comme conséquent, de tâches et de responsabilités de l'ensemble d'un niveau hiérarchique à un niveau inférieur. Entrent dans cette catégorie les cas où le dirigeant octroie à quelques responsables une latitude certaine dans les opérations courantes ainsi que les cas où une équipe de direction est véritablement instaurée.

Au sein de chacune de ces catégories ont dû alors être sélectionnés les changements de dimension organisationnelle pour lesquels la question de recherche posée prend toute sa pertinence.

\subsubsection{La sélection des cas pertinents}

Il est important de signaler que sont apparus dans nos données des changements de dimension organisationnelle ( 5 cas de formalisation sur 19 et 9 cas de développement de la délégation sur 19) non conformes à la description apportée par les auteurs des modèles de métamorphose et, par là, sans intérêt pour notre travail. En effet, selon la perception des faits qu'ont eue les acteurs, ces transformations du mode d'organisation n'ont pas été engagées après la survenue d'un seuil organisationnel qui aurait imposé une révolution immédiate. Certaines ont été perçues comme s'étant déroulées de façon naturelle et très progressive, le changement pouvant souvent être alors qualifié d' «émergent», d'autres ont été décrites comme ayant été engagées en dehors de l'apparition de dysfonctionnements significatifs et plutôt après la mise en place, pour des raisons commerciales, d'un processus d'obtention d'une norme de qualité, ou encore du fait d'aspirations du dirigeant ${ }^{12}$. Ces cas ont été exclus de la suite de nos traitements.

12. Voir Godener et Paturel (1996). 
En revanche, nous avons retenu tous les cas de changement de dimension organisationnelle décrits comme une transformation radicale, délibérée, décidée dans un contexte réactif, c'est-à-dire à la suite de la détection de dysfonctionnements importants, de l'apparition d'une crise financière ou encore de la prise de conscience de la saturation du personnel et / ou du dirigeant. Seules les entreprises ayant agi dans un tel contexte ont atteint ou approché le seuil que nous cherchons à caractériser.

\subsubsection{La caractérisation des seuils}

Pour les 14 cas de formalisation et 10 cas de développement de la délégation ainsi retenus, nous avons alors procédé au second type de codage préconisé par Strauss et Corbin (1990), à savoir le «codage axial», dans le but d'établir des liens entre les changements de dimension organisationnelle de type réactif et leur contexte de survenue. Des relations hypothétiques ont été formulées à partir de quelques cas, puis soumises à la réalité des autres, comme le préconisent aussi bien Glaser et Strauss (1967) que Huberman et Miles (1991). Tout cas atypique a alors été utilisé pour enrichir le modèle en construction : «Quand un site spécifique diverge du modèle, on doit changer le modèle pour y faire entrer cette information plutôt que d'essayer de trouver de bonnes raisons de gommer l'information "dérangeante". » (Huberman et Miles, 1991)

La mise en œuvre de cette méthodologie, inductive par essence, nous a permis de déceler des « régularités » au sein de l'histoire des 23 entreprises étudiées.

\section{L'existence d'événements catalyseurs prévisibles à court terme}

Nous présentons d'abord les résultats obtenus à travers l'étude du contexte de réalisation de ces changements de dimension (3.1) puis indiquons comment la recherche systématique de contre-exemples permet d'affiner nos conclusions (3.2).

\subsection{Le moment de survenue des seuils organisationnels se caractérise par la proximité temporelle d'autres événements liés à la croissance}

L'étude des cas de changements de dimension consécutifs à la survenue d'un seuil organisationnel a permis de mettre en évidence que, selon la perception qu'en ont les dirigeants interrogés, juste en amont (ou au moment) de la survenue du seuil organisationnel, se situe systématiquement un événement marquant lié à la croissance. Ce résultat rejoint le constat de McPhee (1990) qui souligne que «selon beaucoup d'études [sur le changement], les périodes de transition sont créées par des événements - les événements causent et provoquent ces périodes et servent même de points de rupture ».

Revue internationale P.M.E., vol. 15, nº 1, 2002 


\subsubsection{Les événements identifiés}

Trois catégories distinctes d'événements sont apparues comme précédant de peu ou étant concomitantes à toute apparition de seuil. Il s'agit de : 1) un développement du système d'information de gestion, 2) une extension de capacité et 3) le lancement d'une expansion commerciale. Aucun seuil organisationnel n'est apparu en dehors de la réalisation de l'un ou l'autre de ces événements. En revanche, certains cas de seuil organisationnel sont apparus comme liés simultanément à deux ou même trois types d'événements. Chaque catégorie est composée de situations diverses précisées dans le tableau 5 qui indique également le nombre de cas correspondants.

\subsubsection{Une tentative d'interprétation}

Bien que ces éléments d'interprétation soient à utiliser avec précaution et que la question dépasse notre problématique, il est intéressant de se pencher sur les raisons susceptibles d'expliquer cette relation temporelle systématique. Pour certains de nos répondants, les événements cités sont considérés comme ayant fait l'effet d'un catalyseur et ayant rendu crucial un besoin de formalisation ou de développement de la délégation qui, sans doute, préexistait dans l'entreprise mais dont il était possible jusque-là de s'accommoder. Avec le recul du temps, les dirigeants concernés considèrent qu'ils ont constitué la «cause conjoncturelle ${ }^{13}$ » de la survenue des seuils organisationnels.

Ainsi, dans le cas de la formalisation, l'événement situé légèrement en amont (ou juste au moment) de l'apparition du seuil organisationnel a provoqué de nouveaux besoins qui, ajoutés à la situation préexistante, ont rendu incontournable un changement de dimension.

Nous citerons dans ce sens le cas d'une société dont les différents services, jusque-là géographiquement dispersés autour d'une petite localité, ont été regroupés sur un site unique à la superficie très supérieure à celle des locaux utilisés jusqu'alors. Précédemment, au sein de chaque unité géographique, prévalaient l'informel, l'ajustement mutuel et la convivialité. Entre sites, les flux étaient organisés.

C'est comme une bande de copains qui se trouve dans un petit chalet pour une période donnée. On se débrouille, tout le monde participe aux différentes tâches et suit les différents événements. Par exemple, personne ne surveille particulièrement les enfants mais tout le monde est vigilant. La cuisine se fait sans que n'ait été constituée à

13. Nous reprenons ici la terminologie de Mayer (1992) qui distingue les causes structurelles de la crise, qui constituent son origine profonde, et les causes conjoncturelles qui sont immédiates et provoquent l'étincelle aggravant brutalement les difficultés.

Revue internationale P.M.E., vol. 15, n 1, 2002 
Tableau 5

Trois catégories d'événements en relation temporelle avec la survenue des seuils organisationnels

\begin{tabular}{|c|c|c|c|}
\hline \multirow{2}{*}{$\begin{array}{l}\text { Événements marquants } \\
\text { légèrement antérieurs } \\
\text { à la survenue du seuil } \\
\text { organisationnel }\end{array}$} & \multirow[t]{2}{*}{ Situations précises correspondantes } & \multicolumn{2}{|c|}{$\begin{array}{l}\text { Nombre de cas* de corrélation } \\
\text { temporelle avec un seuil de: }\end{array}$} \\
\hline & & Formalisation & Délégation \\
\hline $\begin{array}{l}\text { Le développement du } \\
\text { système d'information } \\
\text { de gestion }\end{array}$ & $\begin{array}{l}\text { - Mise en place ou développement } \\
\text { de l'informatique. } \\
\text { - Adoption d'un système de GPAO. } \\
\text { - Développement de fonctions } \\
\text { administratives jusque-là } \\
\text { simplifiées ou sous-traitées. } \\
\text { - Instauration d'une comptabilité } \\
\text { analytique. }\end{array}$ & 8 cas & 4 cas \\
\hline $\begin{array}{l}\text { Une extension de } \\
\text { capacité }\end{array}$ & $\begin{array}{l}\text { - Automatisation massive de } \\
\text { l'équipement de production. } \\
\text { - Substitution d'une chaîne de } \\
\text { production à une organisation } \\
\text { artisanale. } \\
\text { - Instauration d'un fonctionnement } \\
\text { en deux ou trois postes de huit } \\
\text { heures par jour. }\end{array}$ & 7 cas & 6 cas \\
\hline $\begin{array}{l}\text { Une expansion } \\
\text { commerciale }\end{array}$ & $\begin{array}{l}\text { - Transition d'un marché régional } \\
\text { vers un marché national. } \\
\text { - Transition d'un marché national } \\
\text { vers un marché international. } \\
\text { - Adjonction d'au moins une } \\
\text { activité supplémentaire dans } \\
\text { l'entreprise monoactivité. } \\
\text { - Développement important du } \\
\text { nombre de références. }\end{array}$ & 3 cas & 3 cas \\
\hline
\end{tabular}

* Certains cas de survenue de seuils organisationnels ont été consécutifs à la survenue, peu de temps auparavant, de plusieurs événements marquants. Le total des nombres indiqués dans ce tableau est donc supérieur au total annoncé de cas d'apparition de seuils de formalisation (14) et de développement de la délégation (10).

l'avance une équipe avec un responsable identifié. Mais si les mêmes se trouvent logés sur une très grande surface, la dynamique est cassée et il faut répartir les tâches et organiser les choses.

Le problème principal a été celui de la circulation des produits dans l'entreprise où sont apparus de gros goulots d'étranglement. En outre, les nouveaux locaux offrant plus de place pour le stockage, ceux-ci se sont développés de façon anarchique et spontanée ( «quand on n'a qu'un petit placard, on entasse et on a tout ce qu'il faut en quantité suffisante ; mais quand on a un grand placard, on le remplit ») et les frais financiers s'en sont vite ressentis. 
Un autre exemple est celui de deux des entreprises étudiées qui n'avaient pas prévu, lors de la mise en place d'outils informatiques destinés respectivement à fiabiliser la gestion des stocks et à accélérer l'élaboration des plannings de production, qu'il leur faudrait définir les rôles de chacun à travers des procédures détaillées prévoyant jusqu'aux situations exceptionnelles. Goulots d'étranglement, ruptures de stock s'en sont suivis avant que tout ne soit pensé et organisé formellement.

Le dernier exemple indiqué ici dans le cadre du seuil de formalisation sera celui d'une entreprise, passée en quelques mois d'un marché régional au marché national. Ce cas se caractérise par le fait qu'il n'y a pas eu seulement extension de la zone de chalandise, mais aussi changement brutal de clientèle, puisque, à des garagistes régionaux, l'entreprise a décidé de substituer des grossistes présents sur le plan national afin d'alléger l'effort commercial. Après une période de profonds cafouillages ont été élaborés argumentaire de ventes, tarifs, format des offres commerciales, suivi formalisé de la clientèle et du traitement des commandes, etc. La structure des services commerciaux de l'entreprise en a été profondément transformée. Le rôle de chacun a été défini, ainsi que des procédures permettant de gérer les relations avec la clientèle.

Dans le cas du développement de la délégation, c'est plutôt le temps nécessaire à la préparation de l'événement qui a rendu caduc le mode d'organisation en vigueur dans certaines entreprises.

Ainsi, le système d'information d'une firme étant apparu à organiser et à informatiser, le chef d'entreprise, plus attiré par la recherche constante de l'innovation technique que par l'organisation de sa société, a préféré, pour ce projet, faire confiance aux responsables de fonction dont il s'était précédemment entouré mais qui, jusqu'alors, avaient été cantonnés à l'exécution des tâches quotidiennes. Ayant ainsi pu faire leurs preuves, les cadres concernés ont été, par la suite, associés à l'ensemble des décisions de la firme.

Le plus souvent, le partage des tâches se fait en sens contraire. Le manque de temps pousse le dirigeant à accroître, dans les faits, les responsabilités de quelques-uns sur les opérations quotidiennes, tandis qu'il se consacre essentiellement à un projet en cours (d'extension de capacité, de lancement d'un nouveau produit, de mise en place d'un nouveau d'information, etc.). «J'ai enfin réussi à structurer, à avoir les hommes compétents. Cela m'enlève un grand poids. »

Ainsi, d'après le récit qu'en font a posteriori certains dirigeants d'entreprise ayant géré des changements de dimension organisationnelle, la survenue des seuils organisationnels serait accélérée par un ou plusieurs événements d'une autre nature - tel que le développement du système d'information, l'extension de capacité ou l'expansion commerciale -, qui perturberait l'équilibre précaire de la firme en 
croissance. Pour autant, tous nos répondants n'ont pas établi de lien de cause à effet, se contentant alors de noter une concomitance entre les faits. Les tableaux 6 et 7 synthétisent les dires des répondants.

Au-delà de ces trois événements, un quatrième changement de dimension mérite d'être évoqué dans le cadre de l'étude des changements de dimension organisationnelle : il s'agit de l'ouverture partielle ou totale du capital de l'entreprise, jusque-là familial, à de nouveaux investisseurs. Sur les 12 cas (correspondant à 9 entreprises) d'ouverture du capital à de nouveaux partenaires ou d'achat total par une société extérieure, il n'a été enregistré aucune survenue d'un seuil organisationnel immédiatement après l'événement; aucun investisseur n'a imposé d'emblée un changement d'organisation radical ${ }^{14}$. Cependant, une relation différée est apparue dans deux cas où le nouvel investisseur a imposé la mise en place d'une comptabilité analytique pour l'un et de tableaux de bord pour l'autre. Le mode de fonctionnement initial, fondé sur les savoir-faire individuels et l'ajustement mutuel, s'est révélé incompatible avec la production des informations requises. Alors que leur production représentait un effort jugé considérable par la filiale, les informations fournies à la maison mère sont apparues comme insatisfaisantes et ont considérablement perturbé les relations entre les deux entités jusqu'à ce qu'un véritable processus d'informatisation soit engagé... processus évoqué ci-dessus pour avoir provoqué de profonds dysfonctionnements en matière de gestion de production avant qu'un processus de formalisation de l'entreprise et de développement de la délégation ne soit engagé.

C'est donc en quelque sorte indirectement et seulement lorsque le nouvel investisseur a exigé la remontée régulière d'une quantité importante d'informations que l'ouverture du capital s'est montrée, dans notre terrain d'observation, susceptible d'accélérer la survenue d'un seuil organisationnel.

À ce stade de notre analyse, il apparaît que tous les événements identifiés comme ayant un lien immédiat avec la survenue de seuils organisationnels (développement du système d'information, extension de capacité, expansion commerciale), s'ils étaient connus à l'avance, pourraient servir d'indicateurs à la survenue probable, à court terme, d'un seuil organisationnel... à condition que la réalisation de chacun de ces événements soit systématiquement suivie de la survenue d'un seuil à franchir. Or, il apparaît dans les informations collectées des cas de réalisation de l'un ou l'autre de ces événements qui, selon les dirigeants interrogés, n'ont pas

14. Le fait qu'aucun investisseur n'ait imposé directement un changement de dimension organisationnelle peut être lié au fait qu'aucune entreprise étudiée n'a ouvert son capital dans une période de crise. N'étant pas le dos au mur, les propriétaires-dirigeants d'origine de la firme ont «choisi » des repreneurs dont le projet était d'accompagner leur société en croissance plutôt que de la révolutionner d'emblée.

Revue internationale P.M.E., vol. 15, n ${ }^{\circ} 1,2002$ 


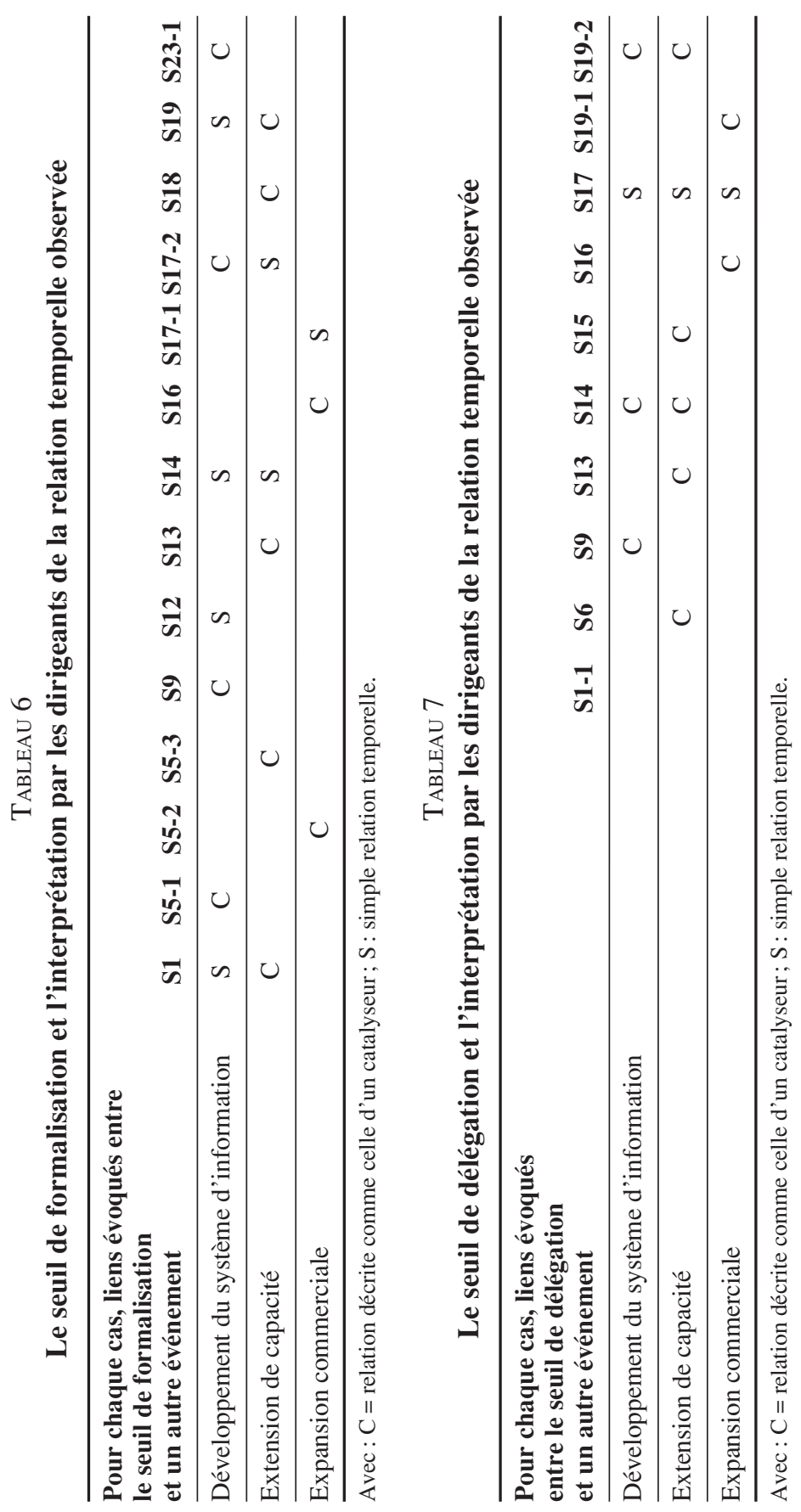

Revue internationale P.M.E., vol. 15, $\mathrm{n}^{\circ}$ 1, 2002 
correspondu à la prise de conscience d'un besoin de formalisation ou de développement de la délégation. Nous avons donc cherché à utiliser ces contre-exemples pour enrichir le modèle en cours de construction en précisant les conditions qui président à la relation étudiée.

À ce stade de notre raisonnement, la question posée devient donc celle-ci : sous quelle(s) condition(s) la réalisation d'un événement de type « développement du système d'information de gestion », «extension de capacité » ou « expansion commerciale » correspond-elle généralement à la survenue d'un seuil organisationnel?

\subsection{La régularité de la relation de proximité temporelle étudiée}

L'usage du « codage axial » nous a permis d'identifier plusieurs conditions susceptibles d'intervenir dans la relation temporelle qui nous intéresse.

En premier lieu, la relation étudiée dépend, et c'est une évidence, du mode d'organisation de l'entreprise au moment où se présente l'événement étudié. Une entreprise à la structure formelle, tout comme une entreprise déjà dotée d'une équipe de direction, ne rencontre pas de seuil de formalisation ou de délégation. Seules sont susceptibles d'être concernées les entreprises n'ayant pas atteint ces stades de développement au moment de la réalisation de l'événement considéré.

Cela étant posé, nous distinguerons les événements relevant des catégories « développement du système d'information » et «extension de la capacité de production», de ceux relevant de «l'expansion commerciale».

Pour les premiers, il apparaît que seuls les événements ayant constitué une mutation radicale et ayant représenté une rupture dans la vie de l'entreprise ont été suivis de la survenue d'un seuil de formalisation et/ou de délégation. Ainsi, lorsque le système d'information a été développé de façon très partielle (un cas) ou de façon très progressive (sur une période supérieure à un an) avec une adjonction régulière de petits modules (deux cas), les dirigeants concernés ont ressenti le besoin d'une évolution parallèle du mode d'organisation, mais cette évolution s'est faite naturellement, sans rupture et donc sans qu'on puisse considérer qu'un seuil organisationnel soit apparu. Il en est allé de même dans deux cas où l'extension de capacité s'est faite à travers la mise en service progressive de surfaces supplémentaires sans que les installations utilisées précédemment ne soient sujettes à modification (p. ex., installation d'une ligne de production s'ajoutant à la ligne de production existante). Dans ces deux cas, l'extension de capacité a ainsi représenté une évolution progressive, une adaptation, plutôt qu'une « révolution» et n'a pas eu d'implication au niveau organisationnel.

Revue internationale P.M.E., vol. 15, nº 1, 2002

(C) 2002 - Presses de l'Université du Québec

Édifice Le Delta I, 2875, boul. Laurier, bureau 450, Sainte-Foy, Québec G1V 2M2 • Tél. : (418) 657-4399 - www.puq.uquebec.ca

Tiré de : Revue internationale P.M.E., vol. $15, n^{\circ} 1$, sous la direction de Pierre-André Julien. 
De plus, il apparaît que l'extension de capacité réalisée dans une firme aux effectifs réduits peut ne pas avoir d'incidence particulière sur l'organisation. En attestent trois cas de déménagements qui ont eu lieu lorsque l'entreprise avait moins de 15 personnes.

Dans notre terrain d'observation, ces trois conditions - stade de développement atteint au moment des faits, caractère radical de l'événement qui a représenté une rupture et, enfin, entreprise à effectif suffisant - permettent de distinguer tous les cas de «développement du système d'information » et «d'extension de capacité » ayant correspondu à la survenue d'un seuil organisationnel, des cas où la situation a été contraire.

En ce qui concerne la catégorie «expansion commerciale », la réalité de nos observations est tout autre. Tandis que presque autant de contre-exemples que d'exemples ont pu être recensés, nous n'avons pas été en mesure d'identifier les conditions éventuelles qui augmenteraient la probabilité d'une proximité temporelle entre une opération d'expansion commerciale majeure et la survenue d'un seuil organisationnel. En particulier, les critères de taille ou d'ampleur du changement ne sont pas apparus comme déterminants. Nous nous garderons donc de conclure en la matière.

\section{Conclusion}

La littérature existante permet d'appréhender, grâce à la description des dysfonctionnements qui se manifestent alors, les seuils organisationnels susceptibles d'être rencontrés par les petites et moyennes entreprises en croissance. La recherche que nous avons menée fournit de nouvelles informations en précisant le moment ultime où les dirigeants de notre terrain d'observation, ayant agi de façon réactive et par franchissement de seuil, ont pris conscience du besoin impératif de changer de dimension organisationnelle.

Nous avons en effet mis en évidence que, sur l'échantillon théorique étudié, ce moment clé a systématiquement été caractérisé par la survenue d'au moins un autre événement dont nous avons établi une liste provisoire - développement du système d'information, extension de capacité, expansion commerciale. Réciproquement, nous avons relevé pour les deux premières de ces trois catégories d'événements que chaque fois qu'elles sont apparues dans une entreprise employant plus de 15 à 20 personnes, et qu'elles ont revêtu la forme d'un changement radical et majeur, est survenu le seuil de formalisation pour les entreprises à la structure informelle et / ou un seuil de délégation pour celles n'étant pas encore dotées d'une équipe de direction. Nous en concluons que les événements relevant des catégories « développement du système d'information de gestion » et «extension de capacité » sont susceptibles d'être utilisés comme annonciateurs de la survenue probable d'un seuil organisationnel. Ces faits sont, en effet, prévisibles quelque temps à l'avance 
puisqu'ils relèvent d'une décision du chef d'entreprise et requièrent une préparation. Les événements de la catégorie «expansion commerciale » semblent également entretenir, dans certains cas, des liens étroits avec la survenue de ces moments critiques, mais dans des circonstances plus complexes à déterminer.

Il en ressort qu'il serait opportun de prendre en compte l'éventualité de la survenue d'un seuil organisationnel au moment de la décision de se lancer (ou pas) dans certaines opérations marquantes telles que le développement radical du système d'information ou une extension brutale de la capacité de production ainsi que dans le cadre de leur préparation.

Cependant, compte tenu du caractère exploratoire de notre recherche et de la taille nécessairement restreinte de notre terrain d'observation, nos résultats ne peuvent être considérés que comme provisoires et méritent d'être complétés par une multiplication des observations permettant d'affiner le modèle.

Il est ainsi probable que d'autres événements représentant des ruptures dans la vie de la petite et moyenne entreprise industrielle en croissance soient susceptibles de provoquer l'accélération de la survenue d'un seuil organisationnel. De même, les conditions selon lesquelles ces événements sont annonciateurs d'un crucial besoin de réorganisation peuvent être précisées : en particulier, le rythme de croissance, le niveau de qualification des salariés et du dirigeant, le degré de rentabilité financière, l'intensité de la concurrence sont, parmi tant d'autres, des caractéristiques de l'entreprise susceptibles d'influer sur le moment de survenue des seuils organisationnels. Enfin, seules ont été étudiées des entreprises industrielles en croissance par croissance interne et ayant franchi avec succès les seuils organisationnels rencontrés; de très nombreuses autres situations restent donc à analyser.

Mais la question de la caractérisation des seuils organisationnels mérite également d'être étudiée sous différents angles de vue complémentaires. Nos résultats correspondent à la perception des faits par les dirigeants d'entreprise ayant participé à l'étude, point de vue essentiel puisque, dans une petite et moyenne entreprise, le dirigeant est le décideur. Mais des observateurs externes (consultants, banquiers, investisseurs éventuels, etc.) ou même internes (comme les salariés) sont susceptibles d'avoir une interprétation différente des faits et d'estimer le changement nécessaire de façon plus précoce. Le croisement de différents points de vue relatifs au parcours d'une entreprise donnée permettrait d'enrichir la connaissance relative à la perception des seuils organisationnels et de mieux informer les dirigeants de ce que leurs partenaires éventuels attendent d'eux.

Ainsi, notre recherche constitue un premier pas vers l'élaboration d'un outil d'alerte du dirigeant de l'entreprise en croissance jusqu'ici quelque peu dépourvu pour identifier à l'avance le moment de la survenue probable du prochain seuil organisationnel qu'il devra franchir et, donc, pour s'y préparer. L'enjeu est de taille et les voies de recherche restent nombreuses.

Revue internationale P.M.E., vol. 15, nº 1, 2002 


\section{Bibliographie}

AdIZES, I. (1991), Les cycles de vie de l'entreprise, Éditions d'Organisations, 270 p.

BARTOl, A. et PH. HeRmel (1986), Piloter l'entreprise en mutation : une approche stratégique du changement, Paris, Éditions d'Organisations, 259 p.

BAsire, M. (1976), «La théorie des cinq niveaux », Direction et gestion, $1^{\text {re }}$ partie, $\mathrm{n}^{\mathrm{o}} 2$, p. $11-21,2^{\text {e }}$ partie, $n^{\circ} 3$, p. $11-22,3^{\mathrm{e}}$ partie, $\mathrm{n}^{\mathrm{o}} 4$, p. 13-20.

BRAC DE LA PERRIÈRE, G. (1978), «L'entreprise moyenne dans le marché des capitaux : conclusion d'un colloque », Banque, $\mathrm{n}^{\circ}$ 379, p. 1321-1331.

Brilman, J. (1985), Gestion de crise et redressement d'entreprises, Paris, Hommes et techniques, $296 \mathrm{p}$.

ChurChiLl, N.C. et V.L. LewIS (1983), «Les cinq stades de l'évolution d'une P.M.E. », Harvard l'Expansion, automne, p. 51-63.

DAVIDSSON, P. (1989), «Entrepreneurship and after? A study of growth willingness in small firms », Journal of Business Venturing, vol. 4, no 3, p. 211-226.

Flamholtz, E.G. (1986), How to Make the Transition from an Entrepreneurship to a Professionally Managed Firm, San Francisco, Jossey-Bass, 231 p.

GASSE, Y. et C. CARRIER (1992), Gérer la croissance de sa PME, Montréal, Les Éditions de l'Entrepreneur.

GELINIER, O. et A. GAULTIER (1974), L'avenir des entreprises personnelles et familiales, Suresnes (France), Hommes et techniques, 254 p.

GIRARD, B. (1997), «Pourquoi les PME restent-elles PME? », Annales des Mines : Gérer et comprendre, septembre, p. 33-42.

Glaser, B.G. et A.L. Strauss (1967), The Discovery of Grounded Theory: Strategies for Qualitative Research, Chicago, Aldine, $257 \mathrm{p}$.

GODENER, A. (1996), La survenue des seuils organisationnels dans les petites et moyennes entreprises industrielles en croissance, Thèse en sciences de gestion, Université Pierre-Mendès-France, Grenoble II, École supérieure des affaires, 364 pages.

Godener, A. et R. PATUREL (1996), Les limites théoriques et pratiques des modèles de métamorphose, Cahiers du CERAG, Université Pierre-Mendès-France, Grenoble II.

GREINER, L.E. (1972), «Evolution and revolution as organizations grow », Harvard Business Review, juillet-août, p. 37-46.

GREINER, L.E. (1977), «De l'utilité des crises dans l'entreprise », Harvard l'Expansion, été, p. 22-29.

HOFER, W. et R. CHARAN (1984), «The transition to professional management : mission impossible? », American Journal of Small Business, été, vol. IX, nº 1, p. 1-11.

Huberman, A.M. et M.B. Miles (1991), Analyse des données qualitatives, recueil de nouvelles méthodes, Bruxelles, De Boeck-Wesmael, 480 p.

KALIKA, M. (1985), «L'efficacité des entreprises est-elle liée à leur structure? », Revue française de gestion, janvier-février, p. 93-104.

Revue internationale P.M.E., vol. 15, nº 1, 2002 
LIPPITT, G.L. et W.H. SCHMIDT (1967), « Crisis in a developing organization », Harvard Business Review, novembre-décembre, p. 102-112.

MAYER, P. (1992), «Organisations en crise : une perspective clinique et analytique », Gérer et comprendre, $\mathrm{n}^{\mathrm{0}} 28$, p. 84-95.

MCPHEE, R.D. (1990), «Alternate approaches to integrating longitudinal case studies », Organization Science, vol. 1, n ${ }^{\circ}$ 4, p. 393-405.

Miller, D. et P.H. Friesen (1984), «A longitudinal study of the corporate life cycle», Management Science, vol. 30, n 10, p. 1161-1183.

MONTMORILLON (DE), B. (1989), «La croissance contractuelle », Revue française de gestion, janvier-février, p. 97-106.

Morse, J.M. (1994), «Designing founded qualitative research», dans N.K Denzin et Y.S. Lincoln (dir.), Handbook of Qualitative Research, Londres, Sage Publications, p. $220-235$.

PARENT, J. (1985), Les firmes industrielles, tome 2, 345 p.; tome 3, Paris, Presses universitaires de France, 415 pages.

SAlter, M.S. (1970), « Stages of corporate development », Journal of Business Policy, $\mathrm{n}^{\mathrm{o}} 1$, p. 23-37.

SMith, K.G., T.R. Mitchell et C.E. Summer (1985), « Top level management priorities in different stages of the organizational life », Academy Management Journal, vol. $28, n^{\circ} 4$, p. $799-820$.

STEINMETZ, L.L. (1969), «Critical stages of small business growth, when they occur and how to survive them », Business Horizons, février, p. 29-36.

STRAuss, A. et J. CORBIN (1990), Basics of Qualitative Research : Grounded Theory Procedures and Techniques, Londres, Sage Publications, $270 \mathrm{p}$.

STRAUSS, A. et J. CORBIN (1994), «Grounded theory methodology : an overview », dans N.K. Denzin et Y.S. Lincoln (dir.), Handbook of Qualitative Research, Londres, Sage Publications, p. 273-285.

TASHAKORI, M. (1980), Management Succession, from the Owner-Founder to the Professional President, NewYork, Praeger, 125 p.

VAN DE VEN, A.H. (1987), « Review essay: four requirements for processual analysis », dans A.M. Pettigrew (dir.), The Management of Strategic Change, Oxford, Basil Blackwell, p. 330-341.

WOLFE, J. et C. JACKSON (1987), «Creating models of the strategic decision-making process via participant recall : a free simulation examination », Journal of Management, vol. $13, \mathrm{n}^{\mathrm{o}} 1$, p. 123-134.

Woodward, J. (1965), Industrial Organization: Theory and Practice, Londres, Oxford University Press, 281 p.

Revue internationale P.M.E., vol. 15, n 1, 2002 